\title{
Food-deprivation-induced suppression of pituitary- testicular-axis in the tilapia Oreochromis mossambicus
}

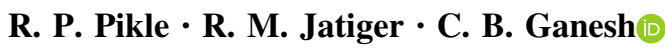

Received: 2 February 2017/Accepted: 15 June 2017/Published online: 26 June 2017

(C) The Author(s) 2017. This article is an open access publication

\begin{abstract}
In the present study, the tilapia Oreochromis mossambicus were exposed to food deprivation for a period of 6 or 12 days and changes in the luteinizing hormone (LH) immunoreactivity in the proximal pars distalis (PPD) of the pituitary gland and the testicular activity were examined. Intensely immunoreactive LH content was noticed in the PPD of the pituitary gland in the initial controls, controls on days 6 and 12, and fasting fish on day 6, whereas the LH immunoreactivity was moderate or weak in fasting fish on day 12 . In addition, although the mean gonadosomatic and hepatosomatic indices among different experimental groups did not show any statistically significant difference, the mean numbers of spermatogonia, spermatocytes, and spermatids were significantly lower in food-deprived fish on days 6 or 12 compared to those of controls. The inhibition of the spermatogenesis was accompanied by the presence of abundant spermatozoa in the lumen of seminiferous tubules of the testis in food-deprived fish, whereas the occurrence of spermatozoa was relatively infrequent in initial controls and controls. Furthermore, refeeding to food-deprived fish on day 6 onwards resulted in occurrence of few intensely stained LH secreting cells and significantly higher numbers of spermatocytes and spermatids concomitant with sparse spermatozoa in majority of tubules compared to those of food-deprived fish. These results suggest that prolonged exposure to food-deprivation causes suppression of the LH secretory activity in the pituitary gland and disruption in the spermatogenesis in O. mossambicus.
\end{abstract}

Keywords Starvation - Luteinizing hormone $\cdot$ Testis $\cdot$ Spermatogenesis $\cdot$ Fish reproduction - Oreochromis mossambicus

\section{Introduction}

Nutrition influences growth, reproduction, and health of fish (Jobling 2015). While availability of food is a great limiting factor, food-deprivation has diverse effects on tissue and plasma components that can interfere with various physiological functions (Mendez and Wieser 1993). Most fishes undergo natural period of fooddeprivation throughout a normal life cycle (Silverstein et al. 1998; Grone et al. 2012; Jobling 2015). Several studies have examined the effect of food deprivation/starvation on reproduction in fish. For example, limitation in feeding causes reduction in the growth rate and delays the spawning time in the European seabass Dicentrachus labrax (Cerda et al. 1994), whereas food-deprivation due to mouthbrooding substantially suppresses ovarian development and androgen and estrogen levels in the African cichlid fish, Astatotilapia burtoni (Grone et al. 2012). On the other hand, exposure to starvation for a period of 40 days at $20{ }^{\circ} \mathrm{C}$ induces

R. P. Pikle · R. M. Jatiger · C. B. Ganesh $(\bowtie)$

Neuroendocrinology Research Lab, Department of Studies in Zoology, Karnatak University, Dharwad 580003 , India

e-mail: ganeshkcd@gmail.com 
testicular regression, but does not affect the onset of recrudescence in the estuarine gobiid fish Gillichthys mirabilis (De Vlaming 1971). Likewise, a significant decrease in the gonadosomatic index (GSI) is observed following food deprivation in the Atlantic cod Gadus morhua (Dutil et al. 2003), whereas starvation with no intermittent feeding causes a significant reduction in the testicular development and maturation in the iteroparous Arctic charr Salvelinus alpinus (Rice 1999). Similarly, feed restriction affects the testicular activity in a phase-dependent manner in the catfish Clarias gariepinus (Suchiang and Gupta 2011). Although there has been extensive studies on the relationship between nutritional status and reproductive health of fishes, majority of these are limited to seasonal breeders except for A. burtoni (Grone et al. 2012) in which suppression of sex steroid hormones levels is observed following starvation due to mouthbrooding. However, duration-dependent effects of food deprivation on luteinizing hormone (LH) secretion and testicular activity in non-seasonally breeding fish are not known. The tilapia Oreochromis mossambicus is a continuous breeder that shows spermatogenic cycle throughout the year (Dadzie 1969). Since many fish are exposed to natural period of starvation at certain times during aquacultural practices (Jobling 2015), the understanding of the effect of food-deprivation and refeeding on reproductive activity of the fish is imperative to obtain maximum benefits. The objective of the present investigation is to determine whether exposure to short- and long-term fooddeprivation affects the LH secretion and the testicular activity in O. mossambicus, and if so, whether this effect is restored after refeeding.

\section{Materials and methods}

\section{Experimental procedure}

Adult $O$. mossambicus were collected from ponds around Dharwad (longitude $75^{\circ} 01^{\prime} \mathrm{E}$, latitude $15^{\circ} 27^{\prime} \mathrm{N}$ ) and brought to the laboratory. The fish were acclimatized to the laboratory conditions 2 months prior to the commencement of experiment. Confirmation of males was done by visual observation of genital openings. The sexual maturity was confirmed by their ability to release milt upon gentle pressing at the ventral surface near the genital opening. The fish weighing $25-35 \mathrm{~g}$ were maintained at a stocking density of six per aquaria (Vol. 70-1; size, $36 \times 12 \times 18$ inches, $\mathrm{L} \times \mathrm{W} \times \mathrm{H}$ ) under natural conditions (photoperiod, $11.33 \pm 0.7$; water temperature, $30.86 \pm 1.02$; dissolved oxygen, $7.16 \pm 0.25$ ). The water was recirculated daily to avoid algal growth and accumulation of planktons that might interfere with the nutritional status of the fish in all aquaria during the period of experimentation.

The experimental setup included six groups ( $n=6$ in each group). The first group of male fish was sacrificed on the day of commencement of experiment and served as the initial controls. The control fish in groups 2 and 3 were fed (Taiyo pet feed, Chennai, India) daily 3 g per aquaria for a period of 6 (controls 6D) and 12 (controls 12D) days, respectively. The fish in groups 4 and 5 were subjected to fasting for 6 (fasting 6D) and 12 (fasting 12D) days, respectively. The fish in sixth group (refeeding group) were food deprived for the first 6 days and then refed with the same quantity of food as in controls up to day 12. The fish in groups 2-6 were sacrificed $(n=30)$. All experimental fish were euthanized following anesthetization with 2-phenoxy ethanol (1:1500, Sigma, USA). At autopsy, the weights of the body, the testis, and the liver were recorded and GSI $(100 \times$ gonad mass/body weight) and hepatosomatic index (HSI; $100 \times$ liver weight/body weight) were calculated.

Histology and morphometry of the testis

The testes were fixed in Bouin's fluid and embedded in paraffin wax after dehydration in ascending series of ethyl alcohol. Five micron-thick serial sections were cut and stained with hematoxylin and eosin. Depending upon the morphology and the size, different spermatogenic cell types were identified. The mean size and characteristics of each spermatogenic cell type are provided in Table 1. Measurements and quantification of different spermatogenic cell types were done using Q capture pro7.1 image analysis system consisting of BX53 microscope attached to a digital CCD color camera (Olympus, Tokyo, Japan). In each testis section, counting of different cell types (spermatogonia, spermatocytes and spermatids) was made from ten randomly chosen tubules. This procedure was done in ten discretely selected sections (each being at least 50 sections 
Table 1 Morphology and size of different spermatogenic cells in the testis of O. mossambicus

\begin{tabular}{llc}
\hline $\begin{array}{l}\text { Spermatogenic cell } \\
\text { type }\end{array}$ & Characteristics & $\begin{array}{l}\text { Cell diameter } \\
\text { (Mean } \pm \text { SE) }\end{array}$ \\
\hline 1. Spermatogonia & $\begin{array}{l}\text { Spherical nucleus, less condensed chromatin, single nucleolus, and faintly } \\
\text { stained cytoplasm }\end{array}$ & $12.00 \pm 0.38 \mu \mathrm{m}$ \\
2. Spermatocytes & Spherical nucleus, deeply condensed chromatin in various meiotic stages & $4.92 \pm 0.20 \mu \mathrm{m}$ \\
3. Spermatid & Indistinct outline with scanty cytoplasm and dense spherical nuclei & $1.75 \pm 0.06 \mu \mathrm{m}$ \\
4. Spermatozoa & Deeply stained spherical head with the flagellum, located in lumen & $1.39 \pm 0.04 \mu \mathrm{m}$ \\
\hline
\end{tabular}

away) to avoid repeated counting of the cells in the same tubule. The total number of each spermatogenic cell type was obtained from 100 tubules/testis/fish. The mean values obtained from each experimental fish $(n=6)$ were finally expressed as mean $\pm \mathrm{SE}$. The density of spermatozoa was determined as sparse or abundant based on the area occupied by the spermatozoa in the lumen.

Immunocytochemistry

The tilapia $O$. mossambicus were perfused transcardially with $20 \mathrm{ml}$ of ice-cold phosphate buffered saline (PBS, pH 7.4) followed by $20-\mathrm{ml}$ ice-cold $4 \%$ paraformaldehyde. The brains were dissected out and post-fixed in the same fixative for $12 \mathrm{~h}$. The tissues were rinsed in PBS and cryoprotected in ice-cold $10 \%(2 \mathrm{~h}), 20 \%$ $(2 \mathrm{~h}$ ), and 30\% (overnight) sucrose solution. Transverse and sagittal frozen sections were cut at $14 \mu \mathrm{m}$ thickness using cryostat (CM1510S; Leica Microsystems, Wetzlar, Germany). For immunocytochemical localization of the LH secreting cells in the proximal pars distalis (PPD) region of the pituitary gland, streptavidin-biotin-peroxidase method was used as described previously (Chabbi and Ganesh 2012, 2015, 2016). Briefly, the sections were rinsed in PBS for $15 \mathrm{~min}$ and incubated with rabbit polyclonal human LH $\beta$ antiserum (National Hormone and Peptide Program, Harbor-UCLA Medical Centre, CA, USA) at 1:8000 dilution for the detection of the LH secreting cells in the PPD region of the pituitary gland. The sections were rinsed in PBS for $10 \mathrm{~min}$ and incubated for $2 \mathrm{~h}$ with biotinylated goat antirabbit immunoglobulin G (Sigma, USA) followed by streptavidin peroxidase (EXTRA 3; Sigma, USA) for $1 \mathrm{~h}$. The sections were again rinsed in PBS and stained with chromogen 3-amino-9-ethyl carbazole to visualize the antigen-antibody complex in the form of a reddish brown precipitate. The sections were rinsed in distilled water and mounted with glycerol-gelatin. The following control procedures were included to verify the specificity of the antibodies: (a) omission of primary antibody, (b) replacement of primary antisera with $1 \%$ BSA, (c) omission of secondary antibody, and (d) preadsorption of diluted primary antibody for LH with either salmon pituitary gland LH or human pituitary gland LH (Sigma, USA) at $10^{-5} \mathrm{M}$ for $24 \mathrm{~h}$ prior to incubation. These control procedures completely blocked the immunoreactions, confirming the specificity of the antibody.

The photographs of the sections of pituitary gland were adjusted for brightness and contrast using PHOTOSHOP CS5, version 12.0 (Adobe Systems Inc, San Jose, CA, USA). The immunoreactivity was evaluated using the Image J software version 1.45 (NIH, Bethesda, MD, USA) as described previously (Chabbi and Ganesh 2015, 2016). Briefly, the pixel intensities were measured in both non-immunoreactive background and immunoreactive content. The threshold pixel intensity of the background was subtracted from the total intensity. The threshold intensity of the immunoreactive cells was measured in five sections of each pituitary gland $(n=6)$ and expressed as integrated units in parentheses (mean \pm SE) under results.

\section{Statistical analysis}

The mean values of spermatogenic cell type/tubule diameter were obtained from 100 tubules of each testis of experimental fish. The data on these morphometric parameters and GSI and HSI were computed from six fish belonging to each experimental group and expressed as mean \pm SE. All parameters were analyzed by oneway analysis of variance (ANOVA) and post hoc Student-Newman-Keuls multiple comparison procedure using SigmaStat 3.5 software. Significant differences for all comparisons were considered at $P<0.05$ level. 


\section{Results}

The mean GSI and HSI did not show any significant difference among different experimental groups (Fig. 1a, b). Histological examinations of the testis in the initial control and control fish revealed the presence of several germ cell cysts that occur along the circumference of the seminiferous tubules (Fig. 2b, c). These cysts consisted of different spermatogenic cell types such as spermatogonia, spermatocytes, spermatids, and spermatozoa (Fig. 2a-c). The spermatogonia were large round cells with faint cytoplasm located close to the periphery of testicular lobules (Fig. 2b). The nucleus was large, spherical, and contained poorly condensed chromatin and a darkly stained small nucleolus in it (Fig. 2b). The spermatocytes were smaller than spermatogonia and had spherical nuclei with deeply stained condensed chromatin in various meiotic stages (Fig. 2b). The spermatids were smaller than spermatocytes and located close to the lumen of the tubule. These cells had indistinct outline with scanty cytoplasm and dense spherical nuclei (Fig. 2b). The spermatozoa were the smallest of the spermatogenic lineage with deeply stained round heads and found in the tubular lumen without any arrangement (Fig. 2a, b).

The mean number of spermatogonia, spermatocytes, and spermatids did not differ significantly among the initial controls and controls 6D and 12D groups (Fig. 3a-c). On the other hand, the seminiferous tubules in the testis of food-deprived fish showed fewer spermatogenic stages (Fig. 2d-f). There was a significant decrease in the number of spermatogonia, spermatocytes, and spermatids in fasting 6D and 12D groups compared to those of initial controls and controls (Fig. 3a-c). The testis in fasting 6D group was characterized by enlarged seminiferous tubules filled with much greater number of sperms concomitant with relative lack of germinal epithelium, spermatogonia, spermatocytes, and spermatids in majority of the tubules (Fig. 2d). The tubule border was still intact in fasting 6D group similar to that of controls 6D group (Fig. 3c). Although large seminiferous tubules were filled with spermatozoa, severe degeneration, collapsing, and necrotic changes in

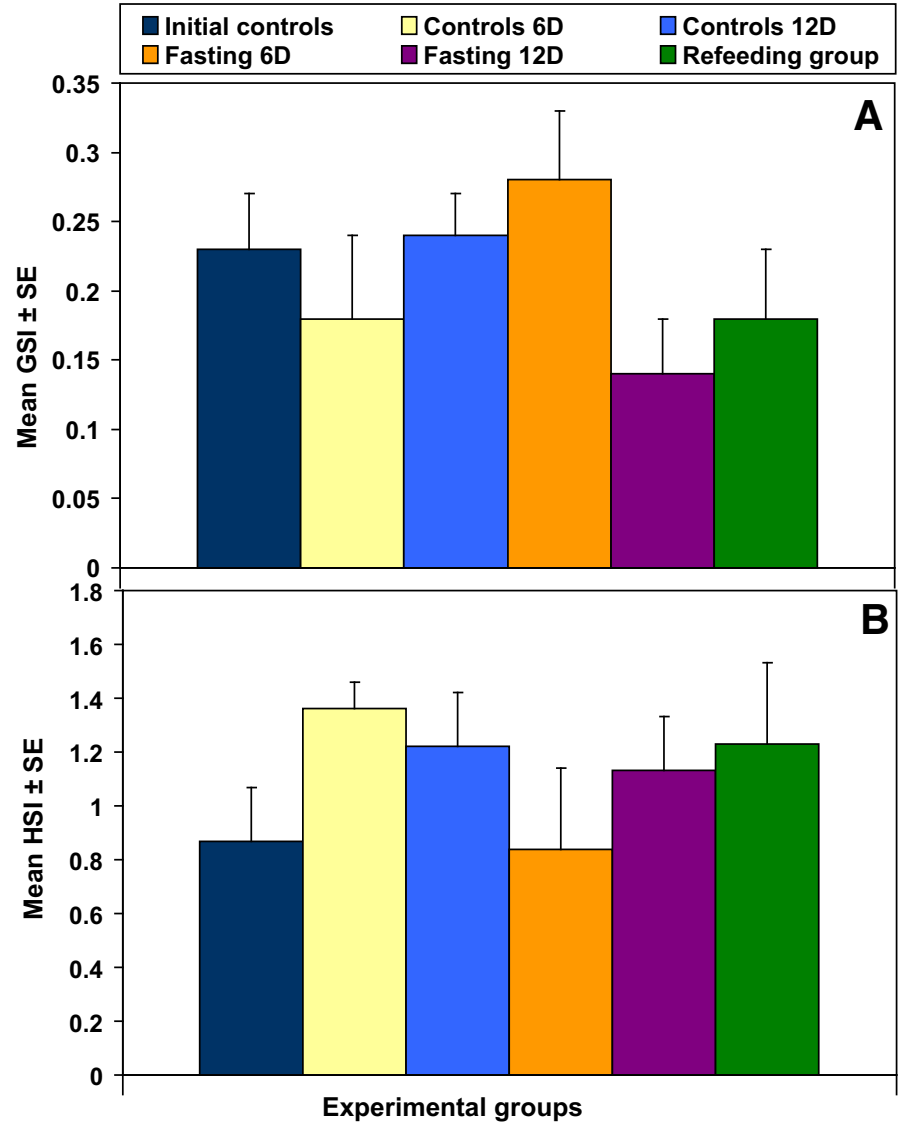

Fig. 1 a and $\mathbf{b}$ Effect of food-deprivation on GSI (a) and HSI (b) in O. mossambicus. Values are mean \pm SE. One-way ANOVA followed by post hoc Student-Newman-Keuls multiple comparison test: no statistically significant differences were noticed among different experimental groups 


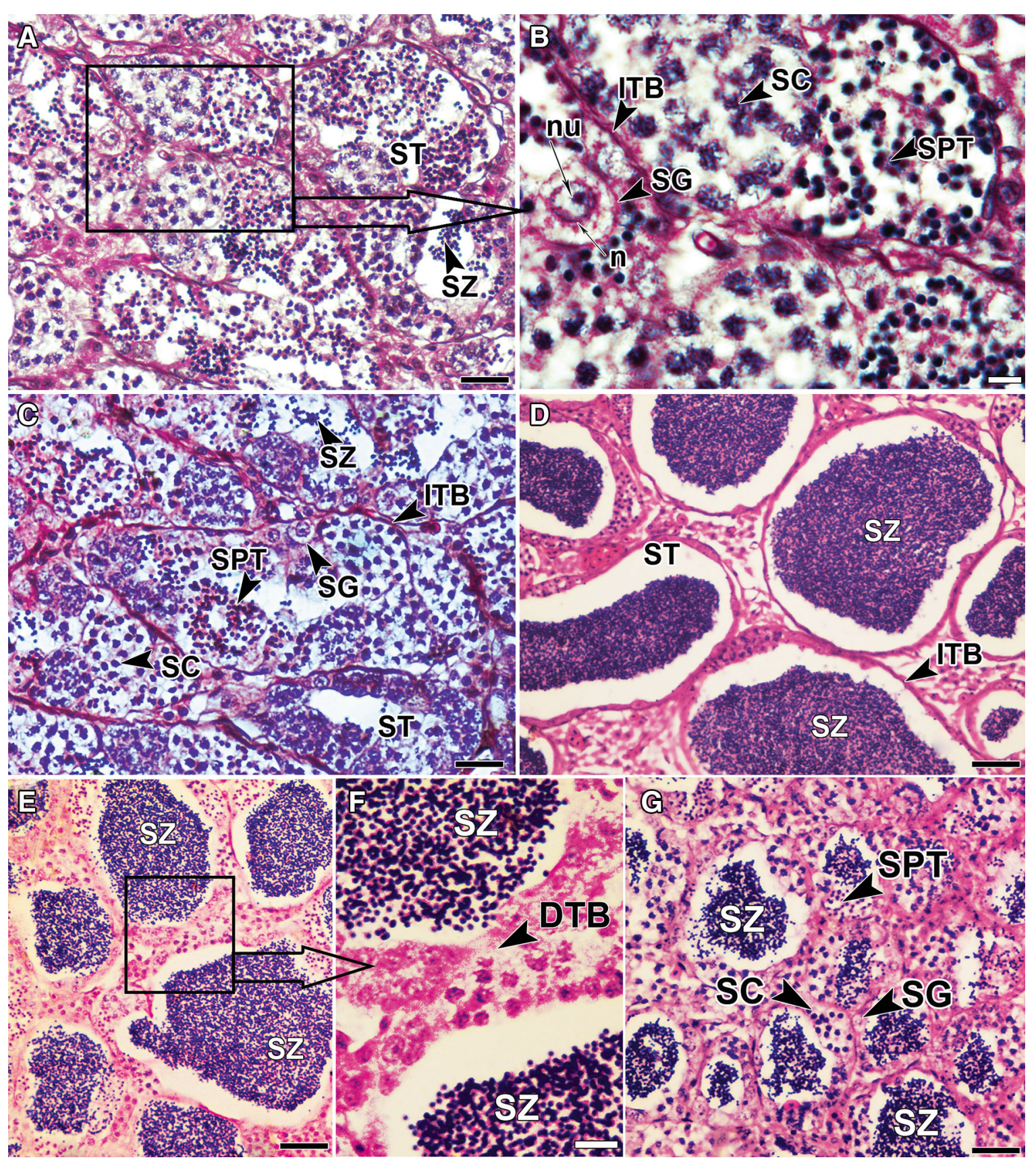

Fig. 2 Cross sections of the testis of $O$. mossambicus showing seminiferous tubules (ST) containing spermatogonia (SG), spermatocyte (SC), spermatids (SPT), and spermatozoa (SZ). Note the presence of abundant spermatozoa in fasting fish on days 6 (d) and 12 (e) compared to sparse spermatozoa and intact tubule border (ITB) in the initial controls (a and b) and controls 6D or 12D groups (c). These changes are partially restored in refeeding group (g). Haematoxylin and Eosin; Scale bar, a, c-e, and g $100 \mu \mathrm{m} ; \mathbf{b} 10 \mu \mathrm{m}$; f $25 \mu \mathrm{m}$. DTB distorted tubule border, $c y$ germ cyst, $n$ nucleus, $n u$ nucleolus

the germinal epithelium of the seminiferous tubules were associated with lack of spermatogenic cell types and distorted tubule border in fasting 12D group (Fig. 2e, f) compared to those of controls 12D group (Fig. 3c).

The fish in refeeding group showed tendency towards the reestablishment of the histoarchitecture of the testis as shown by the appearance of cysts along the borders of tubules and the presence of different spermatogenic cell types (Fig. 2g). Refeeding to food-deprived fish did not improve the number of spermatogonia, whereas the number of spermatocytes and spermatids increased significantly compared to those of fasting $6 \mathrm{D}$ or 12D groups (Fig. 3a-c). Although the mean number of spermatocytes in refeeding group was almost closer to that of controls, the mean number of spermatids was significantly lower compared to those of initial controls 


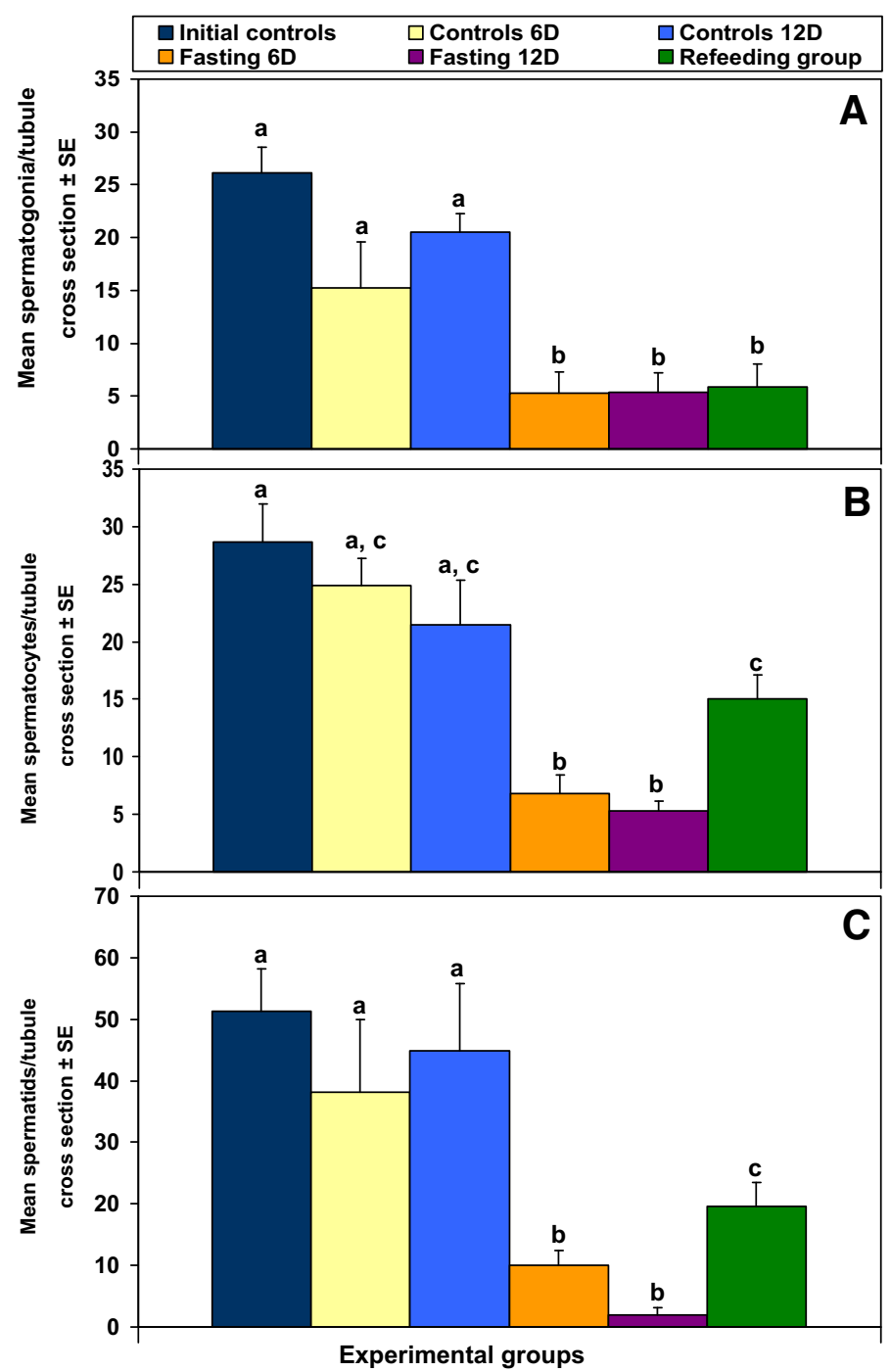

Fig. 3 Effect of food-deprivation on spermatogonia (a), spermatocytes (b) and spermatids (c) in O. mossambicus. Values are mean \pm SE. One-way ANOVA followed by post hoc Student-Newman-Keuls multiple comparison test: Groups with same superscripts are not significantly different from each other, whereas different superscripts indicate significant $(P<0.05)$ difference

and controls (Fig. 3b, c). The mean tubule diameter did not significantly differ among different experimental groups (Fig. 4).

The LH immunostaining was intense in the initial controls $(91.00 \pm 0.94$; Fig. 5a) and controls $6 \mathrm{D}$ or 12D groups $(96.00 \pm 1.34$; Fig. 5b). Exposure of fish to fasting until day 6 also showed the LH immunoreactivity similar to that of initial controls and controls $(92.20 \pm 1.04$; Fig. 5c), whereas the LH immunoreactivity was moderate or weak in fasting fish on day $12(50.58 \pm 1.08$; Fig. 5d) compared to those of initial controls and controls. Refeeding to the fish exposed to the initial food-deprivation, resulted in some intensely stained LH immunoreactive content in the PPD region of the pituitary gland $(95.00 \pm 1.65$; Fig. 5e). However, the extent of immunoreactive area covered in the PPD region was not similar to that of initial controls or controls.

\section{Discussion}

A tight relationship between food availability and reproduction has been observed in fish (Schneider 2004). For example, reduction in feeding rate has been shown to inhibit gonadal maturation in the female Atlantic 


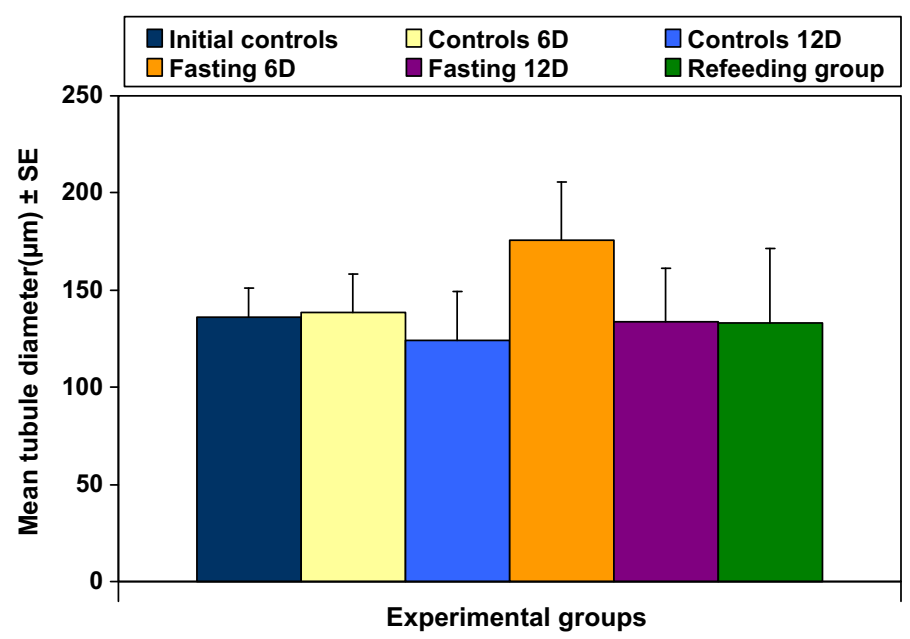

Fig. 4 Effect of food-deprivation on tubule diameter of the testis in O. mossambicus. Values are mean \pm SE. One-way ANOVA followed by post hoc Student-Newman-Keuls multiple comparison test: No statistically significant differences were noticed among different experimental groups

salmon Salmo salar (Thorpe et al. 1990; Reimers et al. 1993), whereas prolonged fasting caused reduction in the weights of the body and the ovary and increased the appearance of atretic follicles in immature female Coho salmon Oncorhynchus kisutch (Yamamoto et al. 2011). Likewise, reduction in the plasma levels of estradiol and the size of eggs as well as the larvae were observed following 6 months of broodstock feeding at a half feed ration in the European seabass D. labrax (Cerda et al. 1994). Whereas exposure of adolescent turbot Scophthalmus maximus to low rations during vitellogenesis affected gonadal maturation (Bromley et al. 2000), the GSI was low in the Atlantic cod G. morhua subjected to food deprivation for a period of 84 days (Dutil et al. 2003). While the majority of these studies point to the vulnerability of the reproductive success following food-deprivation in females, limited studies have revealed inhibitory effect of food-deprivation in males. Restriction in spring time feeding for 2-3 months has been shown to affect maturation in the male Chinook salmon O. tshawytscha (Hopkins and Unwin 1997), whereas 3 weeks of partial and full feed restriction markedly reduced the testicular activity in the catfish C. gariepinus (Suchiang and Gupta 2011). Similarly, starvation with no intermittent feeding caused a significant reduction in the testicular development and maturation in the iteroparous Arctic charr S. alpinus (Rice 1999). In the present study, exposure of $O$. mossambicus to food-deprivation did not significantly affect the GSI as well as the HSI in both fasting 6D and 12D groups compared to those of initial controls or controls. However, the present study reveals the negative effect of food-deprivation on the spermatogenetic activity for the first time in a non-seasonally breeding fish O. mossambicus. A significant reduction in the mean number of spermatogonia, spermatocytes, and spermatids associated with distortion in the tubule borders and relative lack of germinal epithelium in food-deprived fish compared to those of controls or initial controls clearly suggest that exposure to food-deprivation causes detrimental effect on the testicular activity.

On the contrary, several studies have also suggested no influence of feed restriction or starvation in both male and female fish. For instance, no significant differences were noticed in egg hatchability between the fed and starved rainbow trout S. gairdneri (Ridelman et al. 1984), whereas the mean diameter of egg size and GSI were not affected following exposure to limited feeding regime for a period of 550 days from first feeding to sexual maturity in the Tilapia zillii (Coward and Bromage 1999). Similarly, reduction in feeding ratio throughout a year (cycles of 3 days fed/2 days unfed) did not affect the gonadal development in both male and female fish Brycon amazonicus (Carvalho 2001). In adolescent male turbot S. maximus, low ration dietary regime did not significantly influence the maturation (Bromley et al. 2000). Likewise, food restriction (1 g food daily/weekly) for 4 months did not affect the testis development in the convict cichlid Cichlasoma nigrofasciatum (Townshend and Wootton 1984). These results appear to be dependent on the experimental conditions, duration or timing of food restriction, and fish species. 


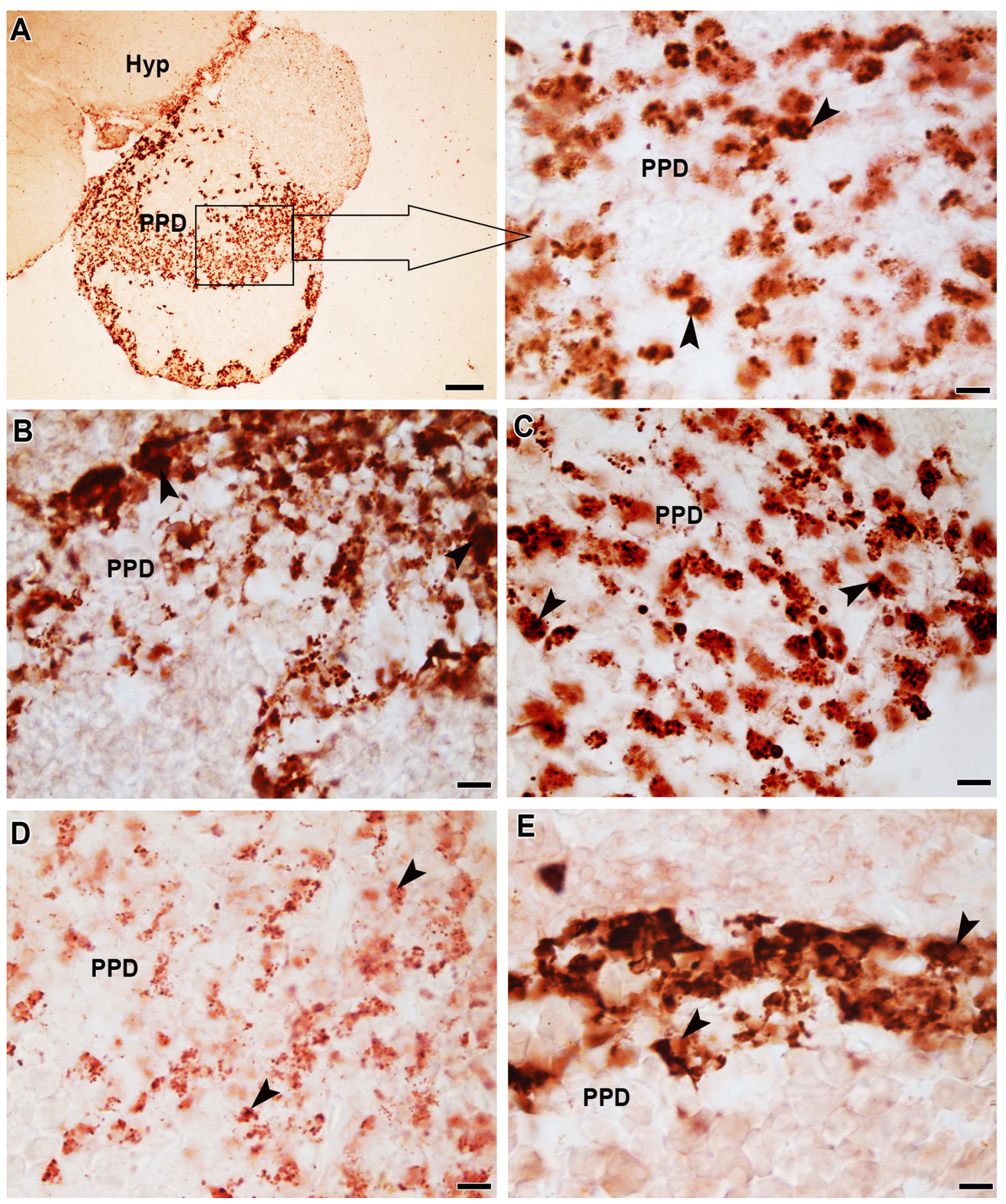

Fig. 5 Effect of food-deprivation on the LH secretory activity in the proximal distalis (PPD) of the pituitary gland in $O$. mossambicus. Note weak or moderate LH immunostaining in transverse sections of the pituitary gland in fish exposed to fasting for 12 days (d) compared to intense LH immunostaining in initial control (a), controls on days 6 or 12 (b), and in fish exposed to fasting on day 6 (c). In refeeding group (e), intensely labeled LH immunoreactive content is seen in the PPD region. Hyp hypothalamus. Scale bar $A 100 \mu \mathrm{m}$; all other figures and magnified view of $A, 25 \mu \mathrm{m}$

In fish, the loss of germinal cells via apoptosis is a normal process throughout the spermatogenesis (Almeida et al. 2008). Franek and Chladkova-Sramkova (1995) demonstrated a close relationship between apoptosis and nutrition in mammalian hybridoma cells. They found artificial starvation due to saline containing medium-induced apoptosis in vitro, whereas refeeding with an amino acid mixture suppressed the 
starvation-induced apoptosis in the cultured hybridoma cells. Escobar et al. (2014) observed an increased apoptosis in germ cells of the testis following exposure of the sea bass D. labrux to food restriction for a period of 9 months. The present study also reveals an inhibition of spermatogenesis in the testis of fish exposed to food-deprivation as shown by a significantly lower number of spermatogonia, spermatocytes, and a relatively lower number of spermatids compared to those of controls. Although it is plausible that the proliferation of spermatogonia from the germinal epithelium was affected, the possibility of loss of spermatogenic cells via food deprivation-induced apoptosis cannot be ruled out. Further studies are required to address this issue.

Sasayama and Takahashi (1972) noticed the presence of large amount of spermatozoa in the lumen surrounded by thin-walled testicular lobules retaining only a small number of spermatogonia in the goldfish Carassius auratus exposed to starvation. In the present study, the organization of germ cell cysts was severely disturbed in fasting fish on day 6 or 12, whereas the integrity of tubule border was lost in fasting fish on day 12 as compared to the presence of intact tubule border in fasting 6D and control fish groups. These changes suggest a duration-dependent detrimental effect of food deprivation on tubule organization in the testis. Furthermore, exposure of the tilapia to food-deprivation for 6 or 12 days resulted in accumulations of abundant spermatozoa compared to the presence of relatively sparse spermatozoa the lumen of the testis in initial controls and controls. Taken together, sparsely found spermatogenic cell types and germ cysts within the intact tubule border concomitant with lumens filled with spermatozoa in fasting 6D group suggest the possibility of rapid completion of spermatogenesis and maturation of spermatids under food-deprivation condition. However, similar changes accompanied by the distortion of tubule border on day 12 of fasting indicate the probability of onset of apoptosis leading to loss of spermatogenic cells and damage to germinal epithelium. Further studies involving the use of apoptotic markers would address this issue.

We may also recall that refeeding at ad libitum in the present study, improved the spermatogenesis as shown by the significantly higher numbers of spermatocytes and spermatids compared to those of fooddeprived fish concomitant with occurrence of sparse spermatozoa and germ cysts in the majority of tubules. While these results confirm that the inhibition of spermatogenetic process is induced by food-deprivation in the present study, the reestablishment of germ cell cysts in tubules of the testis in refed fish might be due to the migration of spermatogonial cells into the cysts from a permanent germinal layer.

In teleosts, the testicular activity is primarily controlled by the pituitary gonadotrophic hormones (GtHs) such as GtH-1 (follicle stimulating hormone, FSH) and GtH-II (LH), which bind to specific receptors in the testis to regulate spermatogenesis and steroidogenesis (Yaron et al. 2003). Kawauchi et al. (1989) reported an increase in the number of GtH-II cells in the pituitary gland at the time of final maturation in teleosts. The expression of the GtH genes was not affected by food restriction in mature female goldfish (Sohn et al. 1998), whereas when food-restricted rats were given unrestricted access to food, the frequency of LH and growth hormone $(\mathrm{GH})$ pulses and the mean levels of FSH increased significantly within 2 days (Sisk and Bronson 1986). Even at the hypothalamic level, although 1 week of food restriction did not result in alterations in the gonadotrophin-releasing hormone $(\mathrm{GnRH})$ neuronal system, food restriction for 2-3 weeks was associated with increased GnRH-immunoreactive neuronal soma size and numbers as well as an increase in fibre intensity in the main fibre pathway to the median eminence in male prairie vole Microtus ochrogaster (Kriegsfeld et al. 2001). These results suggest a stimulatory influence of starvation on hypothalamo-pituitarygonad (HPG) axis. Furthermore, although the regulation of GtH release is under the control of several factors in teleosts, potential influence of neuropeptide Y (NPY) is implicated (Kah et al. 1989). Stimulatory effect of NPY on LH secretion was more pronounced in fasted fish (Cerda-Reverter et al. 1999). Such a possibility cannot be excluded for the present study as exposure of O. mossambicus to 6-day food-deprivation did not affect the LH secretory activity, whereas the LH immunolabeling was moderate or diminished in fish exposed to food-deprivation for 12 days. The duration-dependent variation in LH immunoreactivity might be due to prolonged fasting status and/or involvement of multiple hormones in regulation of reproductive axis. For example, exposure to food-deprivation suppressed the plasma T4 and T3 levels and reduced thyroid tissue sensitivity to thyroid stimulating hormone in fish (Cerda-Reverter et al. 1996). Indeed, suppression of plasma T4 and T3 levels in catfish C. gariepinus maintained under partial and full feed restriction were associated with reduced spermatogenesis (Suchiang and Gupta 2011). Since the thyroid hormones have been shown to modulate the HPG axis in teleosts (Swapna and Senthilkumaran 2007), the dwindling germinal cysts and spermatogenic cell types associated with abundance of spermatozoa in fasting fish 6D group as well as decreased LH immunoreactivity in fasting fish 12D group might be due to down-regulation of hypothalamo- 
pituitary-thyroid axis following starvation-induced energy crisis. However, this possibility needs to be further confirmed in the tilapia. In addition, in the present study, a tendency for reinstatement of pituitary-testicular activity following refeeding apparently substantiates the negative effects of prolonged food-deprivation along the reproductive axis in $O$. mossambicus.

In conclusion, the present study suggests that exposure to food-deprivation for prolonged duration results in deleterious effects on reproduction, which might lead to impairment in the reproductive success of $O$. mossambicus. Although, the short-term exposure to fasting, perhaps, allows the fish to complete the reproductive process under food scarcity conditions, at later stages of food-deprivation, suppression of LH secretory activity in the pituitary gland and disruption in the spermatogenesis are observed. These results have potential implications in aquaculture practices as production of quality gametes is extremely important to achieve reproductive success.

Acknowledgements The work was supported by a grant from UGC-SAP (No. 18/2015/DSA-I), New Delhi. The infrastructural facility received under research grant (No. SR/S0/AS-52/2013) from Science \& Engineering Research Board, Department of Science and Technology (SERB-DST), New Delhi, Govt. of India is gratefully acknowledged.

Open Access This article is distributed under the terms of the Creative Commons Attribution 4.0 International License (http:// creativecommons.org/licenses/by/4.0/), which permits unrestricted use, distribution, and reproduction in any medium, provided you give appropriate credit to the original author(s) and the source, provide a link to the Creative Commons license, and indicate if changes were made.

\section{References}

Almeida FFL, Kristofferson C, Taranger GL, Schulz RW (2008) Spermatogenesis in Atlantic Cod (Gadus morhua): a novel model of cystic germ cell development. Biol Reprod 78:27-34

Bromley JP, Ravier C, Withames PR (2000) The influence of feeding regime on sexual maturation, fecundity and atresia in firsttime spawning turbot. J Fish Biol 56:264-278

Carvalho EG (2001) Restricao alimentar e reproducao em peixes. Jaboticabal: Centro de Aquicultura da UNESP. PhD thesis

Cerda J, Carrillo M, Zanuy S, Ramos J (1994) Effect of food ration on estrogen and vitellogenin plasma levels, fecundity and larval survival in captive sea bass, Dicentrarchus labrax: preliminary observations. Aquat Living Resour 7:255-266

Cerda-Reverter JM, Zanuy S, Carrillo M, Kah O (1996) Development of enzyme immunoassays for 3,5,3'-triiodo-L-thyronine and L-thyroxine: time-course studies on the effect of food deprivation on plasma thyroid hormones in two marine teleosts, sea bass (Dicentrarchus labrax L.) and sea bream (Sparus aurata L.). Gen Comp Endocrinol 103:290-300

Cerda-Reverter JM, Sorbera LA, Carrillo M, Zanuy S (1999) Energetic dependence of NPY-induced LH secretion in a teleost fish (Dicentrarchus labrax). Am J Physiol 277:R1627-R1634

Chabbi A, Ganesh CB (2012) Stress-induced inhibition of recruitment of ovarian follicles for vitellogenic growth and interruption of spawning cycle in the fish Oreochromis mossambicus. Fish Physiol Biochem 38:1521-1532

Chabbi A, Ganesh CB (2015) Evidence for the Involvement of dopamine in stress-induced suppression of reproduction in the cichlid fish Oreochromis mossambicus. J Neuroendocrinol 27:343-356. doi:10.1111/jne.12269

Chabbi A, Ganesh CB (2016) Neuroanatomical evidence for the involvement of $\beta$-endorphin during reproductive stress response in the fish Oreochromis mossambicus. J Chem Neuroanat 77:161-168

Coward K, Bromage NR (1999) Spawning frequency, fecundity, egg size and ovarian histology in groups of Tilapia zillii maintained upon two distinct food ration sizes from first-feeding to sexual maturity. Aquat Living Resour 12:11-22

Dadzie S (1969) Spermatogenesis and the stages of maturation in the male cichlid fish Tilapia mossambica. J Zool 159:399-403

De Vlaming VL (1971) The effects of food deprivation and salinity changes on reproductive function in the estuarine Gobiid fish (Gillichthys mirabilis). Biol Bull 141:458-471

Dutil JD, Lambert Y, Chabot D (2003) Winter and spring changes in condition factor and energy reserves of wild cod compared with changes observed during food-deprivation in the laboratory. ICES J Mar Sci 60:780-786

Escobar S, Felip A, Salah M, Zanuy S, Carrillo M (2014) Long-term feeding restriction in prepubertal male sea bass (Dicentrarchus labrax L.) increases the number of apoptotic cells in the testis and affects the onset of puberty and certain reproductive parameters. Aquaculture 433:504-512

Franek F, Chladkova-Sramkova K (1995) Apoptosis and nutrition: involvement of amino acid transport system in repression of hybridoma cell death. Cytotechnology 18:113-117

Grone BP, Carpenter RE, Lee M, Maruska KP, Fernald RD (2012) Food deprivation explains effects of mouthbrooding on ovaries and steroid hormone, but not brain neuropeptide and receptor mRNAs in an African Cichlid fish (Astatotilapia burtoni). Horm Behav 62:18-26

Hopkins CL, Unwin MJ (1997) The effect of restricted spring time feeding on growth and maturation of freshwater-reared chinook salmon, Oncorhynchus tshawytscha (Walbaum). Aquacult Res 28:545-549

Jobling M (2015) Fish nutrition research: past, present and future. Aquacult Int 24:767-786

Kah O, Pontet A, Danger JM, Dubourg P, Pelletier G, Vaudry H, Calas A (1989) Characterization, cerebral distribution and gonadotropin release activity of neuropeptide Y (NPY) in the goldfish. Fish Physiol Biochem 7:69-76 
Kawauchi H, Suzuki K, Itoh H, Swanson P, Naito N, Nagahama Y, Nozaki M, Nakai Y, Itoh S (1989) The duality of teleost gonadotropins. Fish Physiol Biochem 7:29-38. doi:10.1007/BF00004687

Kriegsfeld LJ, Ranalli NJ, Trasy AG, Nelson RJ (2001) Food restriction affects the gonadotropin releasing hormone neuronal system of male prairie voles (Microtus ochrogaster). J Neuroendocrinol 13:791-798

Mendez G, Wieser W (1993) Metabolic responses to food deprivation and refeeding in juveniles of Rutilus rutilus (Teleostei Cyprinidae). Environ Biol Fish 36:73-81

Reimers E, Kiorrefjord AG, Stavostrand SM (1993) Compensatory growth and reduced maturation in second sea winter farmed Atlantic salmon following starvation in February and March. J Fish Biol 43:805-810

Rice CD (1999) Restricted feeding, spermatogenesis and growth in Arctic Charr (Salvelinus alpinus)-The identification of two possible gametogenic control points. Masters thesis, Memorial University of Newfoundland, USA

Ridelman JM, Hardy RW, Brannon EL (1984) The effect of short-term starvation on ovarian development and egg viability in rainbow trout (Salmo gairdneri). Aquaculture 37:133-140

Sasayama Y, Takahashi H (1972) Effect of starvation and unilateral castration in male goldfish (Carassius auratus), and a design of bioassay for fish gonadotropin using starved goldfish. Bull Fac Fish Hokkaido Univ 22:267-279

Schneider JE (2004) Energy balance and reproduction. Physiol Behav 81:289-317

Silverstein J, Breninger TJ, Baskin DG, Plisetskaya EM (1998) Neuropeptide Y-like expression in the salmon brain increases with fasting. Gen Comp Endocrinol 110:157-165

Sisk CL, Bronson FH (1986) Effects of food restriction and restoration on gonadotropin and growth hormone secretion in immature male rats. Biol Reprod 35:554-561

Sohn YC, Yoshiura Y, Kobayashi M, Aida K (1998) Effect of sex steroids on the mRNA levels of gonadotropin I and II subunits in the goldfish Carassius auratus. Fish Sci 64:715-721

Suchiang P, Gupta BBP (2011) Effects of partial and full feed restriction on the plasma levels of thyroid hormones and testicular activity in the male air breathing catfish (Clarias gariepinus) during different phases of breeding cycle. Int J Biol 3:32-42

Swapna I, Senthilkumaran B (2007) Thyroid hormones modulate the hypothalamo-hypophyseal-gonadal axis in teleosts: molecular insights. Fish Physiol Biochem 33:335-345

Thorpe JE, Talbot C, Miles MS, Keay DS (1990) Control of maturation in culture Atlantic salmon, Salmo salar, in pumped seawater tanks, by restricting food intake. Aquaculture 86:315-326

Townshend TJ, Wootton RJ (1984) Effects of food supply on the reproduction of the convict cichlid, Cichlasoma nigrofasciatum. J Fish Biol 24:91-104

Yamamoto Y, Luckenbach JA, Middleton MA, Swanson P (2011) The spatiotemporal expression of multiple coho salmon ovarian connexin genes and their hormonal regulation in vitro during oogenesis. Reprod Biol Endocrinol 9:52. doi:10.1186/1477$7827-9-52$

Yaron Z, Gur G, Melamed P, Rosenfeld H, Elizur A, Levavi-Sivan B (2003) Regulation of fish gonadotropins. Int Rev Cytol 225:131-185 